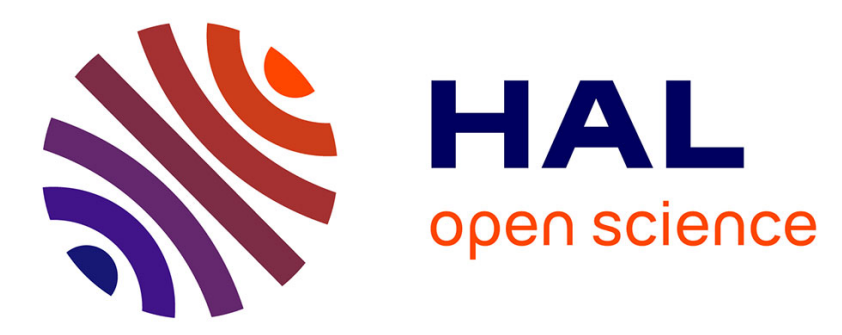

\title{
Dynamics of nonspherical microbubble oscillations above instability threshold
}

Matthieu Guédra, Sarah Cleve, Cyril Mauger, Philippe Blanc-Benon, Claude Inserra

\section{- To cite this version:}

Matthieu Guédra, Sarah Cleve, Cyril Mauger, Philippe Blanc-Benon, Claude Inserra. Dynamics of nonspherical microbubble oscillations above instability threshold. Physical Review E , 2017, 96 (6), pp.063104. 10.1103/PhysRevE.96.063104 . hal-01705578

\section{HAL Id: hal-01705578 \\ https://hal.science/hal-01705578}

Submitted on 9 Feb 2018

HAL is a multi-disciplinary open access archive for the deposit and dissemination of scientific research documents, whether they are published or not. The documents may come from teaching and research institutions in France or abroad, or from public or private research centers.
L'archive ouverte pluridisciplinaire HAL, est destinée au dépôt et à la diffusion de documents scientifiques de niveau recherche, publiés ou non, émanant des établissements d'enseignement et de recherche français ou étrangers, des laboratoires publics ou privés. 


\title{
Dynamics of nonspherical microbubble oscillations above instability threshold
}

\author{
Matthieu Guédra, ${ }^{1, *}$ Sarah Cleve, ${ }^{2}$ Cyril Mauger, ${ }^{2}$ Philippe Blanc-Benon, ${ }^{2}$ and Claude Inserra ${ }^{1}$ \\ ${ }^{1}$ Univ Lyon, Université Claude Bernard Lyon 1, Centre Léon Bérard, INSERM, UMR 1032, LabTAU, F-69003, Lyon, France \\ ${ }^{2}$ Univ Lyon, École Centrale de Lyon, INSA de Lyon, CNRS, LMFA UMR 5509, F-69134 Écully, France
}

(Received 11 September 2017; published 5 December 2017)

\begin{abstract}
Time-resolved dynamics of nonspherical oscillations of micrometer-sized bubbles are captured and analyzed using high-speed imaging. The axisymmetry of the bubble shape is ensured with certainty for the first time from the recordings of two synchronous high-speed cameras located at $90^{\circ}$. The temporal dynamics of finite-amplitude nonspherical oscillations are then analyzed for various acoustic pressures above the instability threshold. The experimental results are compared with recent theories accounting for nonlinearities and mode coupling, highlighting particular effects inherent to these mechanisms (saturation of the instability, triggering of nonparametric shape modes). Finally, the amplitude of the nonspherical oscillations is given as function of the driving pressure both for quadrupolar and octupolar bubbles.
\end{abstract}

DOI: 10.1103/PhysRevE.96.063104

\section{INTRODUCTION}

Nonspherical bubble oscillations arising from the instability of the bubble surface is a largely observed and reported phenomenon. These oscillations are known to be parametrically excited by the acoustically driven spherical oscillations of the bubble, a mechanism similar to the hydrodynamic Faraday waves observed on a vertically oscillating liquid layer [1]. When the driving acoustic pressure overcomes a critical value, the surface instability manifests as the triggering of particular shape modes corresponding to zonal harmonics of the initially spherical bubble. Theories have been derived for many years to describe such a phenomenon. Early theories based on the expansion of the bubble surface perturbation into spherical harmonics and linearization of the fluid dynamical equations led to the well-known parametric equation governing the shape modes amplitude, describing the bubble spherical stability conditions and the main characteristics of the nonspherical oscillations [2-5]. Further developments were conducted to account for viscous dissipation [6-9] and effect of weakly nonlinear spherical oscillations [10] on the spherical stability of the bubble. More recently, theoretical works have been expanded to the nonlinear interactions between vibrational modes [11-15] to account for sound radiation [16,17] and translational instability [18,19] of nonspherical bubbles. In general, nonlinearities coming from mode coupling and involved in the spherical, translational, and shape dynamical equations can be seen as one mechanism allowing saturation of the instability leading to nonspherical oscillations of finite amplitude. Experiments have also been conducted to investigate the conditions of appearance of nonspherical oscillations, leading to several reports of stability diagrams [10,20-23].

However only few of them present direct measurements of the temporal dynamics of shape oscillations. Early works reported such observations on radiation-modulated driven and free damped oscillations of millimetric bubbles [24,25] or drops [25,26] (thus, below the parametric threshold). With the recent and considerable developments of high-speed imaging techniques, similar studies are now possible on

\footnotetext{
*matthieu.guedra@inserm.fr
}

high-frequency-driven micrometer-sized bubbles [23,27,28] and time-resolved dynamics of nonspherical oscillations have been reported over the past few years on coated microbubbles such as ultrasound contrast agents $[29,30]$ or armored bubbles [31,32]. Nevertheless, experiments generally disregard the finite amplitude properties of parametrically excited nonspherical oscillations and direct measurements are rarely compared with the most recent theoretical developments accounting for nonlinear saturation and mode coupling. Moreover, while all theories assume axisymmetry of the bubble shape as a starting point of their mathematical formulation, this hypothesis is generally mentioned as being fulfilled in experiments but has never been examined in details and quantified rigorously. In this paper, we first make use of an experimental setup based on two synchronous high-speed cameras to demonstrate and discuss the axisymmetry of nonspherical bubbles. Then, experiments are conducted to capture the temporal dynamics of the nonspherical oscillations above the parametric instability threshold, highlighting nonlinear, finite-amplitude oscillations predicted by recent models.

\section{EXPERIMENTAL SETUP AND MODAL DECOMPOSITION}

Experiments are conducted on the basis of an original setup described in a previous study [27] whose technical details are briefly recalled here. Single laser-induced air bubbles of few tens of micrometers in size are trapped in a cubic tank of distilled undegassed water by applying a standing-wave ultrasonic field of frequency $f_{a}=31.25 \mathrm{kHz}$. The bubble oscillations are driven by a slowly varying amplitude-modulated acoustic pressure field of the form

$$
p_{a}(t)=P_{a} \cos \left(2 \pi f_{a} t\right)\left[1-\eta \cos \left(2 \pi f_{m} t\right)\right],
$$

where $P_{a}$ is the mean acoustic pressure amplitude, $f_{m} \ll f_{a}$ is the low modulation frequency $\left(f_{m}=78.125 \mathrm{~Hz}\right.$, $\left.f_{a} / f_{m}=400\right)$, and $\eta$ is the modulation amplitude. This modulation allows periodic triggering of the nonspherical oscillations on short time durations [27,28]. Once trapped on a stable position, the bubble oscillations are captured using two synchronous high-speed cameras (Vision Research, Phantom V12.1) located at $90^{\circ}$ (Fig. 1). Both cameras 


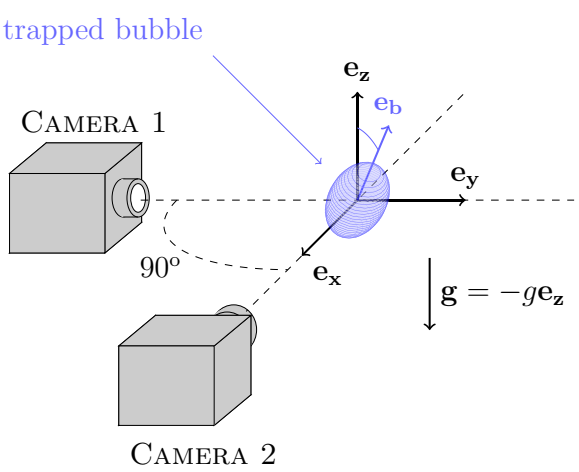

FIG. 1. Schematic view of the optical setup. The axis of symmetry of the nonspherical bubble is identified by the unit vector $\mathbf{e}_{\mathbf{b}}$.

have the vertical $\mathbf{e}_{\mathbf{z}}$ axis as shared axis. We define the $\left(\mathbf{e}_{\mathbf{x}}, \mathbf{e}_{\mathbf{z}}\right)$ plane as the view plane captured by the first camera, and the $\left(\mathbf{e}_{\mathbf{y}}, \mathbf{e}_{\mathbf{z}}\right)$ plane as the view plane captured by the second one. The bubble contour is obtained from backlit illumination using continuous light sources (LED) and its dynamics is recorded with an acquisition rate of 180064 frames per second and a frame size of $128 \times 128$ pixels. The bubble contour extracted from one recorded picture then leads to discrete values of the surface coordinate $r_{s}(\theta, t)$, which can be expanded over the Legendre polynomial basis,

$$
r_{s}(\theta, t)=\sum_{n=0}^{N} a_{n}(t) P_{n}(\cos \theta),
$$

to finally get the modal coefficients $a_{n}$ using the orthogonality property of the polynomials $P_{n}$ :

$$
a_{n}(t)=\frac{2 n+1}{2} \int_{-1}^{1} r_{s} P_{n}(x) d x, \quad \text { with } x=\cos \theta .
$$

The coefficients $a_{n}$ directly correspond to the respective amplitudes of the bubble vibrational modes, including the spherical one $(n=0)$. Two exemplary results of their temporal evolution on one modulation period are presented in Fig. 2, for two bubbles of respective radii $48 \mu \mathrm{m}$ and $70 \mu \mathrm{m}$ mainly exhibiting quadrupolar [Fig. 2(a)] and octupolar [Fig. 2(b)] deformations. Here and throughout the paper, all quantities are dimensionless: the spherical mode coefficient oscillating around the mean radius $R_{0}=\left\langle a_{0}(t)\right\rangle$ is plotted as $a_{0} / R_{0}-1$, while the shape mode coefficients oscillating around a zero mean value are divided by $R_{0}$. The bubble remains spherical at the early and last phases of the modulation corresponding to the lowest values of the instantaneous pressure amplitude [see Eq. (1)]. Due to the modulation, the acoustic pressure dynamically crosses the critical pressure threshold, leading to surface instability and appearance of nonspherical oscillations. The corresponding modal coefficients $a_{2}$ (quadrupolar) and $a_{3}$ (octupolar) present a characteristic exponential growth until reaching a maximum amplitude. Then, because the acoustic pressure falls below the instability threshold during the last phase of the modulation, the nonspherical oscillations are damped until complete disappearance. One can also observe weak oscillations of the fourth mode $a_{4}$ existing for the quadrupolar bubble presented in Fig. 2(a): these oscillations are not induced by the classical parametric instability mechanism but are a signature of nonlinear effects. This will be further commented in Sec. IV.

It is worth noting that expanding the surface coordinate on zonal harmonics according to Eq. (2) implicitly assumes axisymmetry of the bubble shape. In Sec. III, we make use of the recordings from the two synchronous cameras to discuss the symmetry properties of the bubble, which will give confidence in the analysis of the results presented in Sec. IV.

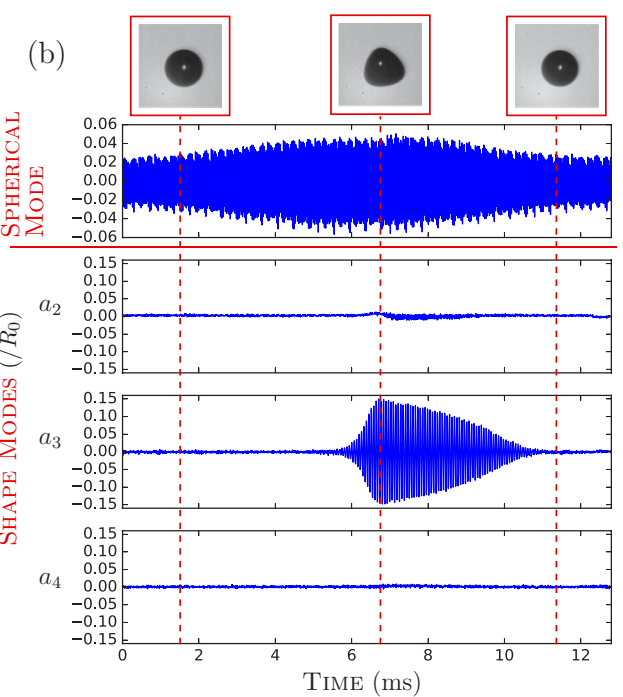

FIG. 2. Temporal evolution of the amplitude of the spherical mode $\left(a_{0}\right)$ and the first three surface modes, for (a) a bubble of radius $48 \mu \mathrm{m}$ exhibiting a quadrupolar deformation, and (b) a bubble of radius $70 \mu \mathrm{m}$ exhibiting a octupolar deformation. All the modal coefficients are dimensionless. The time length corresponds to one period of modulation $f_{m}^{-1}=12.8 \mathrm{~ms}$. (a) Quadrupolar mode. $R_{0}=48 \mu \mathrm{m}, P_{a}=18.4 \mathrm{kPa}$, $\eta=0.38$. (b) Octupolar mode. $R_{0}=70 \mu \mathrm{m}, P_{a}=8.6 \mathrm{kPa}, \eta=0.33$. 
(a)

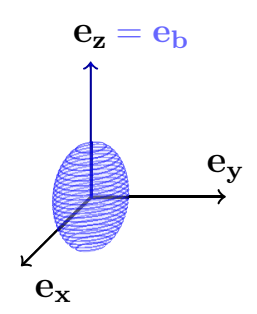

(b)

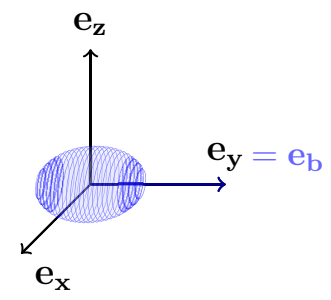

(c)
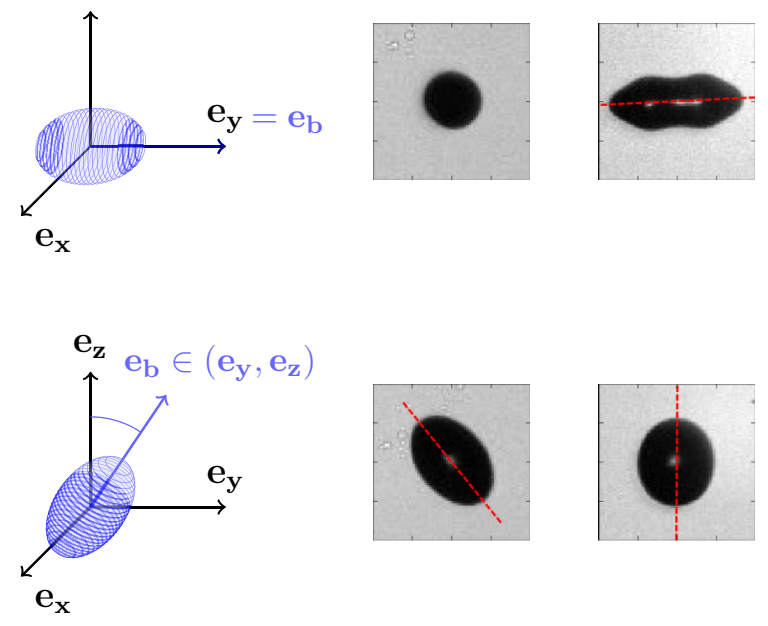

(d)
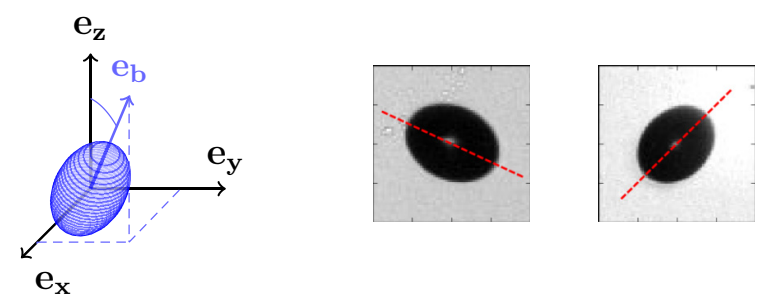

FIG. 3. Bubble orientation and associated side views on both cameras. The axis of symmetry of the nonspherical bubble is identified by the unit vector $\mathbf{e}_{\mathbf{b}}$.

\section{AXISYMMETRY OF SURFACE MODES}

The entire set of experiments-collecting a large number of bubbles of various radii driven at various pressure amplitudes-led us to observe that the symmetry axis of the nonspherical bubble is not fully determined by our experimental conditions (neither by a preferential direction in the acoustic field nor by the gravity [33]) but generally randomly oriented in the 3D space. Therefore, the bubble apparent contour captured by one camera does not necessarily correspond to the surface coordinate and care must be taken when analyzing the coefficients obtained from the modal decomposition. Typical examples of bubble orientations observed in experiments are given in Fig. 3, associated with the corresponding snapshots captured by both cameras. On each snapshot is indicated the axis of symmetry of the bubble apparent contour, i.e., the respective orthogonal projections of $\mathbf{e}_{\mathbf{b}}$ on the $\left(\mathbf{e}_{\mathbf{x}}, \mathbf{e}_{\mathbf{z}}\right)$ plane and $\left(\mathbf{e}_{\mathbf{y}}, \mathbf{e}_{\mathbf{z}}\right)$ plane. The first three cases [Figs. 3(a)-3(c)] correspond to particular orientations of the bubble axis of symmetry $\mathbf{e}_{\mathbf{b}}$, for which information recorded by at least one camera can be used to obtain a proper modal decomposition of the bubble surface.

In the most convenient case, the axis of symmetry of the bubble apparent contour should be oriented in the vertical direction on both cameras [Fig. 3(a)]. In that case, the bubble contour is nearly identical on both cameras and one can assert that the axisymmetry of the bubble is entirely defined by the $\mathbf{e}_{\mathbf{z}}$ axis. Corresponding snapshot series on two acoustic periods are shown in Fig. 4, attesting to the axisymmetry of the bubble by rotation of $90^{\circ}$ around the vertical axis. These snapshot series also clearly illustrate the period doubling usually observed for parametrically excited nonspherical oscillations (first parametric resonance). Performing the modal decomposition of the bubble contour captured by both cameras then leads to the temporal curves shown in Fig. 5. These results confirm that the modal decomposition is invariant to rotation around the vertical axis and allow to ensure that the coefficients well describe the oscillations of the spherical and nonspherical modes. A deeper analysis of the curves, although discussed with more details in the next section, also reveals that for the quadrupolar shaped bubble considered here, the second shape mode oscillates at half the driving frequency (first parametric resonance) while a component mostly oscillating at the driving frequency is also observed on the fourth shape mode (nonlinear effect). The lack of the third mode is also consistent with the expected nonlinear energy transfers predicted by theory (even modes mainly transfer energy towards other even modes [13]).

A second convenient case allows to fully define the axisymmetry of the bubble when the axis of symmetry corresponds either to $\mathbf{e}_{\mathbf{x}}$ or $\mathbf{e}_{\mathbf{y}}$ [Fig. 3(b)]. In that case, the bubble remains apparently spherical on one of the two cameras, while the bubble contour captured by the other view can be directly related to the surface coordinate and expressed by Eq. (2). Corresponding snapshot series on two acoustic periods are shown in Fig. 6, attesting to the axisymmetry of the bubble along the $\mathbf{e}_{\mathbf{y}}$ axis. Similar to the previous case, the
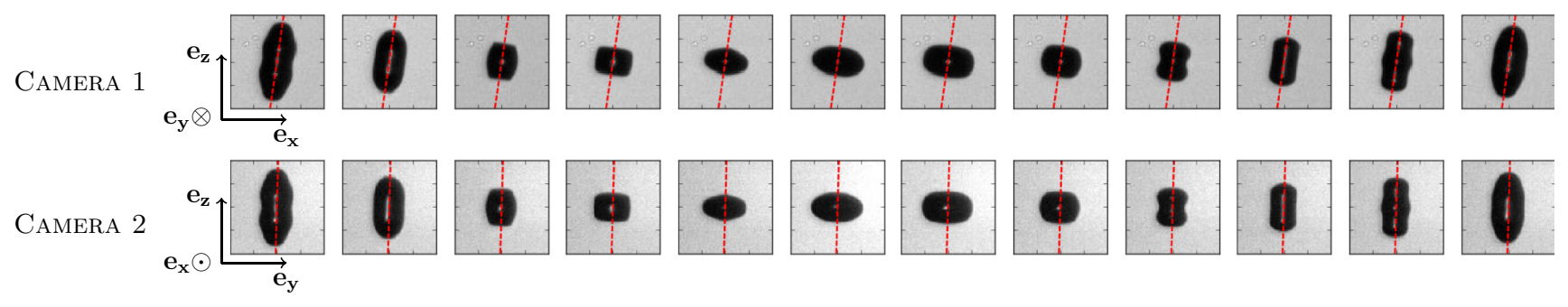

FIG. 4. Snapshot series on two acoustic periods corresponding to case (a) in Fig. 3. Time between two successive snapshots is $5.55 \mu$ s. 


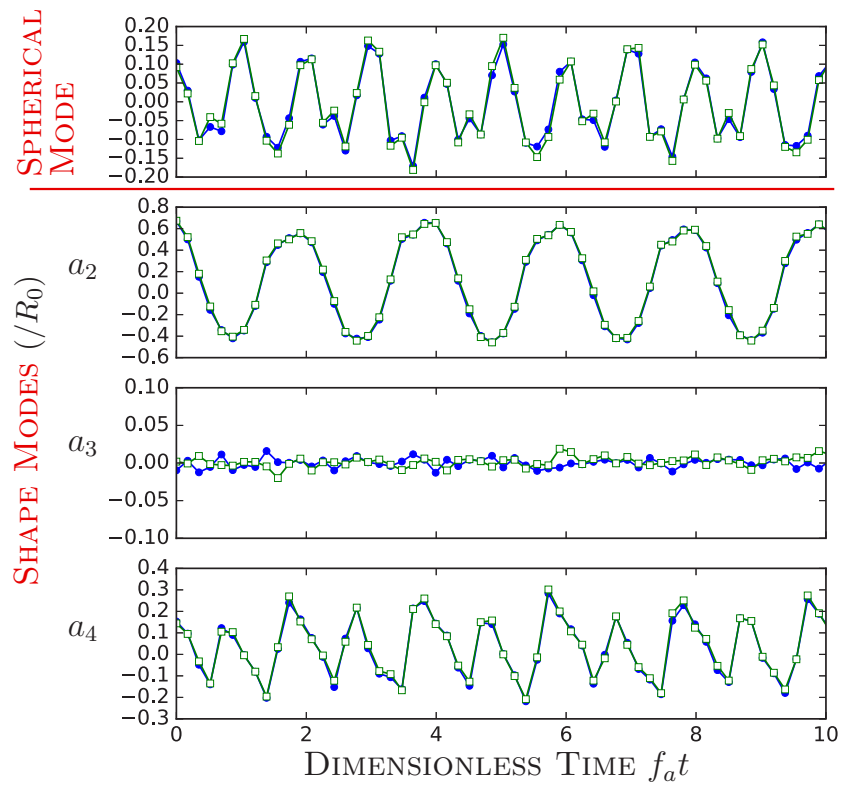

FIG. 5. Temporal evolution of the modal coefficients on ten acoustic periods corresponding to case (a) in Fig. 3, obtained from camera 1 (blue curves with circles) and camera 2 (green curves with squares).

period doubling of the nonspherical oscillations is also clearly observed. Performing the modal decomposition of the bubble contour captured by both cameras then leads to the temporal curves shown in Fig. 7. Observing the results obtained from the first camera directly shows the non-existence of shape modes coefficients because of the apparent sphericity of the bubble, while the results obtained from the second camera are similar to those presented in Fig. 5. It is worth noting that the spherical oscillations obtained on both views show obvious differences since the apparent spherical oscillations obtained on the first camera result from a combination of all vibrational modes. This can be easily demonstrated by considering the modal decomposition obtained from the second camera and computing the surface coordinate Eq. (2) in the particular situation of a $90^{\circ}$ rotation, so that

$$
r_{s}(\pi / 2, t) \simeq a_{0}(t)-\frac{1}{2} a_{2}(t)+\frac{3}{8} a_{4}(t)
$$

reduced here up to the fourth shape mode. The results obtained from Eq. (4) are given by the red solid curve in Fig. 7, which nearly overlays the modal coefficient obtained from the first camera. It should be emphasized at this point that the modal decomposition can naturally lead to inaccurate results, as illustrated here by the apparent spherical oscillations of the bubble.

The exemplary cases presented in Figs. 3(a) and 3(b) correspond to ideally oriented bubbles for which the definition of axisymmetry is unambiguous. Another suitable case is illustrated in Fig. 3(c), for which one axis of symmetry is oriented in the vertical direction on at least one camera. This allows to assert that the axis of symmetry of the bubble $\mathbf{e}_{\mathbf{b}}$ necessarily lies in the view plane of the other camera, thus the bubble contour can be described by Eq. (2) and used for modal decomposition. Finally, in the most general case [Fig. 3(d)] where both apparent axes of symmetry are randomly oriented, bubble contours are purely apparent contours in 3D space and cannot be described by Eq. (2). In that case, the modal decomposition of the surface coordinate leads to artificial modal coefficients and further analysis becomes irrelevant. In the next section, all the results presented correspond to bubbles for which axisymmetry is ensured [cases presented in Figs 3(a)-3(c)].

\section{CHARACTERISTICS OF THE NONSPHERICAL OSCILLATIONS}

\section{A. Summary of the model}

In the following, we further analyze the dynamics of the nonspherical oscillations through direct comparisons with a suitable theory. In a previous study [27], we observed nonlinear mode coupling and the necessity of introducing this effect theoretically was pointed out. The chosen model is based on a set of dynamical equations derived by Doinikov [19] at the second order of accuracy, latter expanded to cubic order by Shaw $[13,14]$. The full equations derived in the above-mentioned references are however strongly simplified in the present study by assuming (i) uniform incident acoustic pressure, (ii) negligible translational motion, (iii) viscous dissipation at the lowest order (boundary layer approximation), and (iv) accuracy to second order. The importance of accounting for viscous dissipation can notably be inferred from an analysis of the Ohnesorge number which demonstrates that viscous effects cannot be neglected for micrometer-sized bubbles and are expected to increase with the order of the shape mode [23]. Retaining these hypotheses, the differential equations governing the spherical mode $a_{0}$ and nonspherical modes

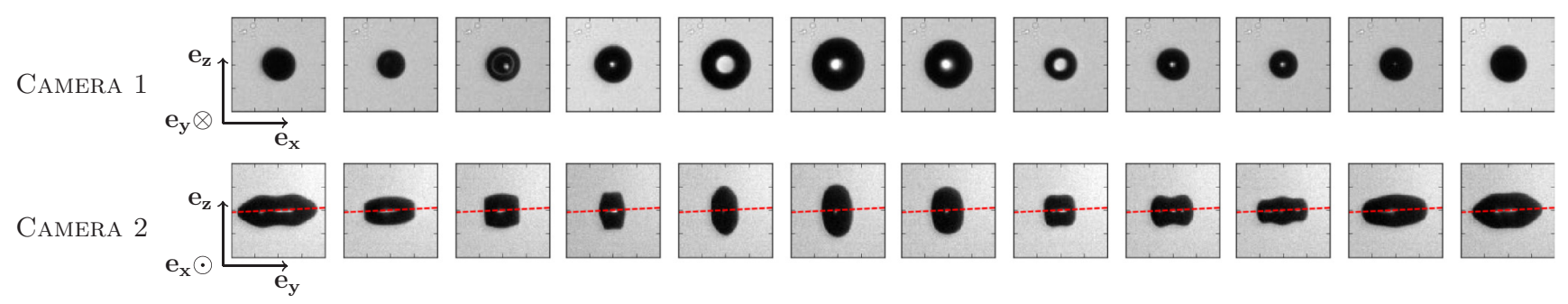

FIG. 6. Snapshot series on two acoustic periods corresponding to case (b) in Fig. 3. Time between two successive snapshots is $5.55 \mu$ s. 


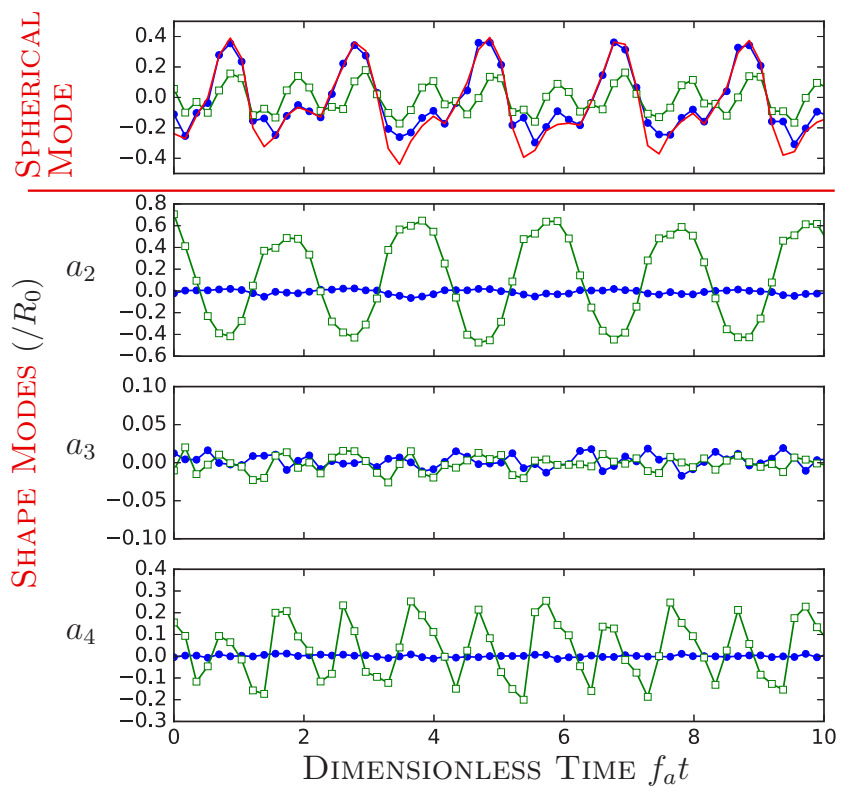

FIG. 7. Temporal evolution of the modal coefficients on ten acoustic periods corresponding to case (b) in Fig. 3, obtained from camera 1 (blue curves with circles) and camera 2 (green curves with squares). The red solid curve on the top graph corresponds to the apparent spherical oscillations computed from camera 2 using Eq. (4).

$a_{n}(n=2,3,4)$ can be summarized as

$$
\begin{aligned}
a_{0} \ddot{a}_{0}+\frac{3}{2} \dot{a}_{0}^{2}= & \frac{1}{\rho}\left(p_{\infty}+\frac{2 \sigma}{R_{0}}\right)\left(\frac{R_{0}}{a_{0}}\right)^{3 \gamma}-\frac{p_{\infty}+p_{a}(t)}{\rho} \\
& -\frac{2 \sigma}{\rho a_{0}}-4 v \frac{\dot{a}_{0}}{a_{0}}+\epsilon^{2} h_{0}\left(a_{i}^{2}, \dot{a}_{i}^{2}, a_{i} \dot{a}_{i}\right), \\
\ddot{a}_{n}+B_{n} \dot{a}_{n}- & A_{n} a_{n}=\epsilon h_{n}\left(a_{i}^{2}, \dot{a}_{i}^{2}, a_{i} \dot{a}_{i}, a_{i} a_{j}, a_{i} \dot{a}_{j}\right),
\end{aligned}
$$

where $\rho$ and $\nu$ are the liquid density and kinematic viscosity, $\sigma$ is the surface tension, $\gamma$ is the gas polytropic index, $p_{\infty}$ and $p_{a}(t)$ are the static part and the acoustic part of the liquid pressure, and $A_{n}$ and $B_{n}$ are the classical time-varying coefficients [7]:

$$
\begin{gathered}
A_{n}(t)=(n-1)\left[\frac{\ddot{a}_{0}}{a_{0}}-\frac{(n+1)(n+2) \sigma}{\rho a_{0}^{3}}\right. \\
\left.-(n+2)\left(1+\frac{2 n \delta}{a_{0}}\right) \frac{2 v \dot{a}_{0}}{a_{0}^{3}}\right], \\
B_{n}(t)=3 \frac{\dot{a}_{0}}{a_{0}}+(n+2)\left[(2 n+1)-(n+2) \frac{2 n \delta}{a_{0}}\right] \frac{2 v}{a_{0}^{2}},
\end{gathered}
$$

where $\delta$ denotes the viscous boundary layer thickness. The functions $h_{0}$ and $h_{n}$ involved in Eqs. (5) and (6) gather all the contributions of the nonlinear interactions between shape modes at the second order of approximation $(i, j=2,3,4)$. Their mathematical expressions are not explicited here for the sake of conciseness but they can be found in Refs. [13,19]. The numerical integration of Eqs. (5) and (6) is achieved for a modulated acoustic pressure field $p_{a}(t)$ of the form given by Eq. (1).

\section{B. Temporal dynamics}

Experimental and theoretical results are compared in Fig. 8 for a bubble of radius $R_{0}=44 \mu \mathrm{m}$ driven at mean acoustic pressure $P_{a}=22.5 \mathrm{kPa}$ and modulation amplitude $\eta=0.23$. It should be emphasized that we make use of the periodicity of the bubble oscillations at the modulation frequency to substantially increase the time resolution from the combination of around ten modulation periods of the entire acquired movie. This allows to get almost 60 experimental points per acoustic period and highly resolved temporal dynamics. Figure 8(a) reveals the onset of the second shape mode with characteristic dynamics including exponential growth of the instability, saturation and slow decay. This nonspherical mode, reaching the largest amplitude values, corresponds to the most unstable

(a)

(b)
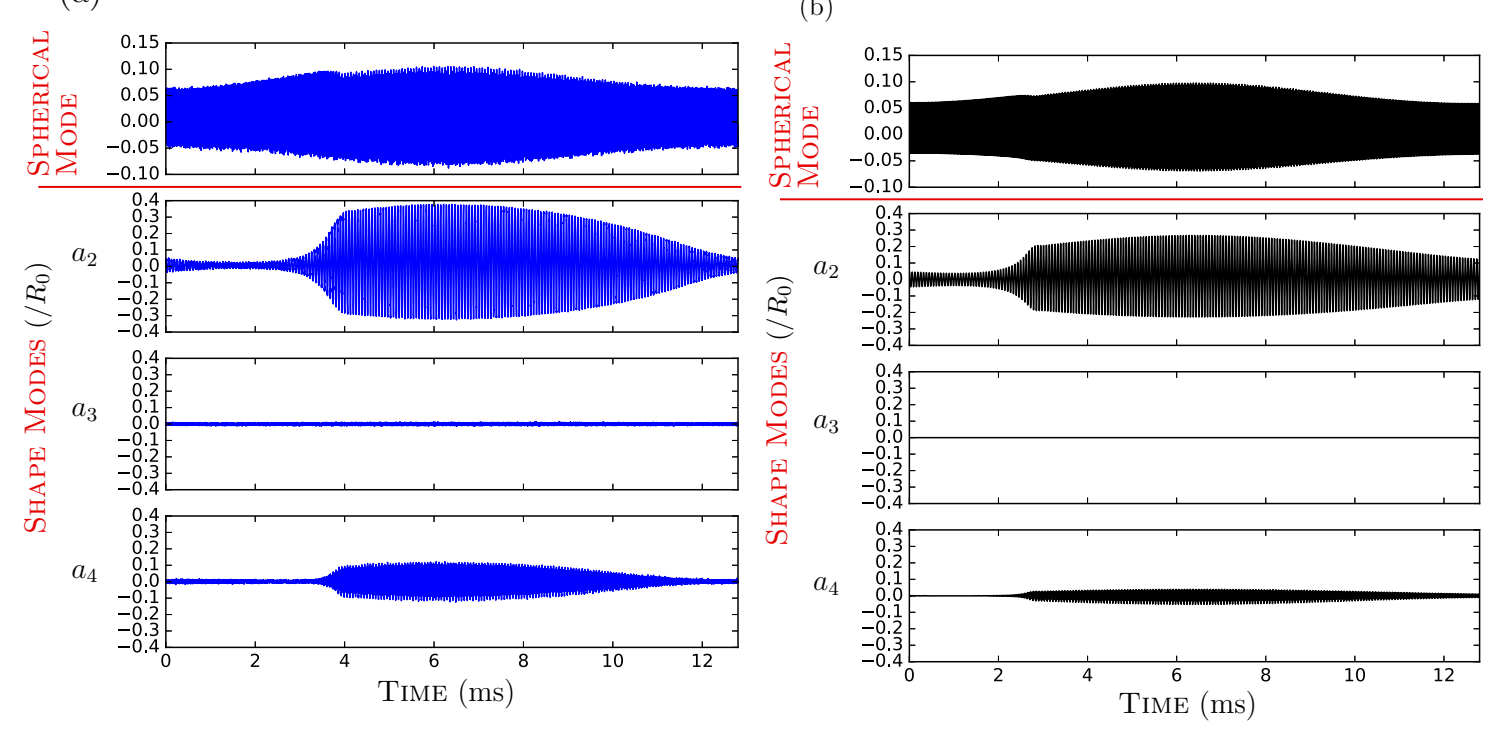

FIG. 8. Temporal evolution of the modal coefficients for a bubble of radius $R_{0}=44 \mu \mathrm{m}$ driven at mean acoustic pressure $P_{a}=22.5 \mathrm{kPa}$ and modulation amplitude $\eta=0.23$. The time length corresponds to one period of modulation $f_{m}^{-1}=12.8$ ms. (a) Experiments. (b) Theory. 


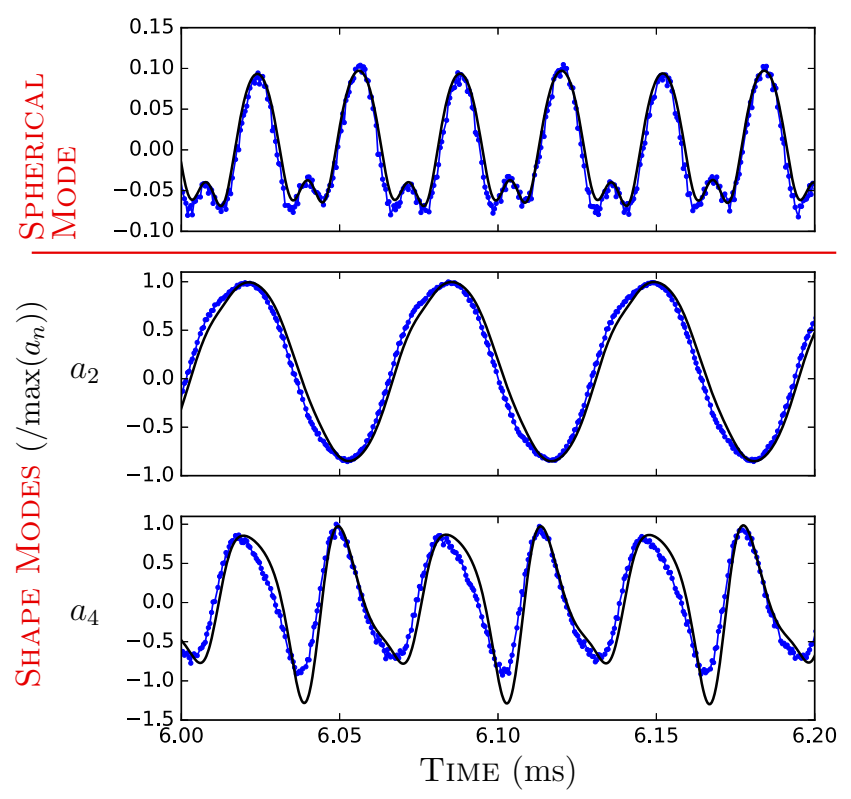

FIG. 9. Comparison between theory (black curves) and experiments (blue curves with circles) at the acoustic time scale.

mode parametrically excited by the spherical oscillations of the bubble. Given the bubble radius $R_{0}=44 \mu \mathrm{m}$ and acoustic frequency $f_{a}=31.25 \mathrm{kHz}$, the computation of the acoustic pressure threshold [7] for this mode gives $P_{2, \text { th }} \simeq 18.1 \mathrm{kPa}$, indeed lower than the mean acoustic pressure $P_{a}=22.5 \mathrm{kPa}$ used in experiments. The experimental results also show the appearance of the fourth shape mode, although computing the pressure threshold for this mode gives $P_{4, \text { th }} \simeq 37 \mathrm{kPa}$. Consequently, this additional mode does not come from a classical parametric excitation but from nonlinear effects, in particular from quadratic contributions of the second mode $a_{2}$ to the dynamics of the fourth mode $a_{4}$. This analysis is

(a)

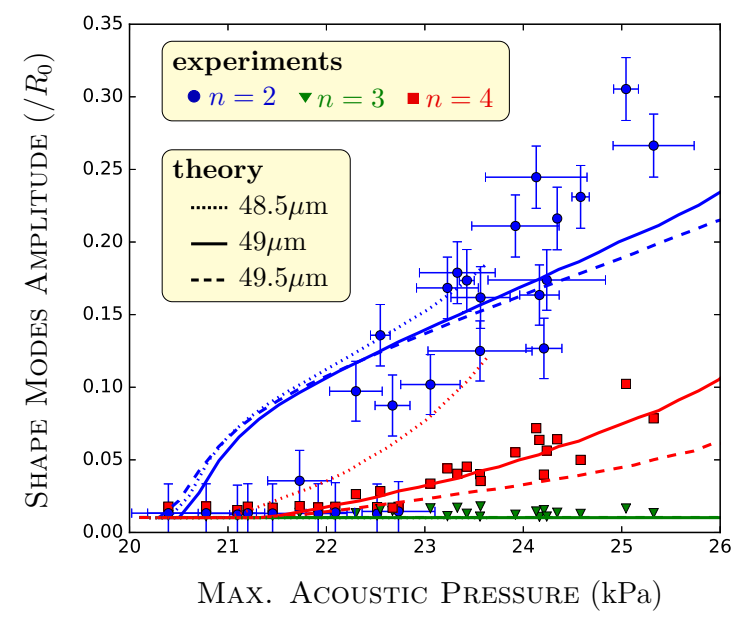

also supported by the numerical simulations in Fig. 8(b), which are in agreement with the experimental observations. It is clearly observed that, whereas the second shape mode is the only unstable mode, the theory predicts oscillations of the fourth shape mode. It is worth noting that the third shape mode does not appear through the same process, which is also consistent with theoretical predictions stating that even modes can only excite other even modes through nonlinear coupling [13]. Experimental and theoretical results are further compared at the acoustic time scale in Fig. 9 by zooming at the middle of the modulation period (the modal coefficient $a_{3}$ has been removed for clarity). Note that while the global dynamics observed in experiments are well captured by the model, a difference remains concerning the amplitude of the oscillations (see Fig. 8). To get a direct comparison of the temporal dynamics, we chose to present the shape modes coefficients normalized by their maximum amplitude. Such a representation has not been made on the spherical oscillations of the bubble and the top graph is a direct zoom-in of Fig. 8: this allows us to highlight the excellent agreement between theory and experiments, including fundamental-driving oscillations, second-harmonic oscillations (nonlinearities of the spherical mode), and (weak but observable) subharmonic oscillations induced by the nonspherical oscillations. A good agreement is also obtained on the temporal dynamics of the nonspherical oscillations. The second shape mode oscillates at half the driving frequency, with a more pronounced distortion observed in experiments. The fourth shape mode oscillates mainly at the driving frequency (quadratic contribution of the second mode) and exhibits strong nonlinear oscillations which are correctly captured by the model.

\section{Shape modes amplitudes}

Collecting several measurement series made on bubbles of different radii driven with increasing pressure amplitudes,

(b)

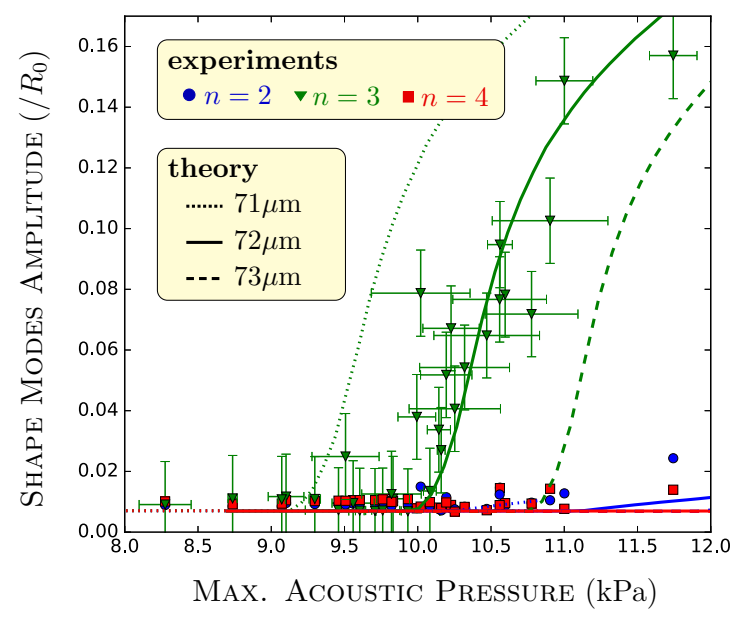

FIG. 10. Shape modes amplitude as function of the acoustic pressure. The experimental results are represented by symbols for $n=2$ (blue circles), $n=3$ (green triangles), and $n=4$ (red squares). The error bars are only provided for the main modes for clarity; however, similar uncertainties are obtained for the other shape modes. The theoretical results are represented by curves with colors corresponding to modal coefficients and line styles corresponding to three values of bubble radius. (a) Quadrupolar bubble. $R_{0} \in$ [46-49] $\mu \mathrm{m}$. (b) Octupolar bubble. $R_{0} \in[70-73] \mu \mathrm{m}$. 
Fig. 10 shows the maximum amplitude of the nonspherical oscillations as function of the acoustic pressure. Figure 10(a) presents results obtained for bubbles of radii lying in [46-49] $\mu \mathrm{m}$ exhibiting mainly quadrupolar deformations, while Figure 10(b) presents results obtained for bubbles of radii lying in [70-73] $\mu \mathrm{m}$ exhibiting mainly octupolar deformations. On both graphs, the maximum amplitude of each shape mode is plotted as function of the maximum acoustic pressure reached during one modulation period, thus $(1+\eta) P_{a}$ according to Eq. (1). In order not to overload the graphs, the uncertainties on experimental data are only provided for the main modes $[n=2$ in Fig. 10(a) and $n=3$ in Fig. 10(b)]; however, similar uncertainties are obtained for the other shape modes. An increase of the shape modes amplitudes is obtained when the acoustic pressure exceeds a critical value, found to be around $22 \mathrm{kPa}$ for the quadrupolar mode and $9.5 \mathrm{kPa}$ for the octupolar mode. In Fig. 10(a), the gradual appearance of the fourth shape mode is also clearly observed, as a consequence of the nonlinear nonspherical oscillations of large amplitudes. Such a nonlinear behavior is, however, not visible in Fig. 10(b) and stronger oscillations driven at larger acoustic pressures would be necessary to possibly observe the excitation of even modes by the third shape mode as predicted by theory [13]. Nevertheless, the theory qualitatively reproduces the observations in the range of acoustic pressures considered in this study, both for parametrically excited shape modes [ $n=2$ in Fig. 10(a) and $n=3$ in Fig. 10(b)] and nonlinearly excited shape modes $[n=4$ in Fig. 10(a)]. Although globally consistent with the experimental results, the numerical simulations also point out the sensitivity of the theory to the bubble static radius, for which a slight change of 1-2 $\mu \mathrm{m}$ can lead to noticeable differences on the nonspherical oscillations. An even better agreement would require better accuracy and control of the experimental bubble radius, while approximate solutions of Eqs. (5) and (6) would be very instructive to capture the general dependency of the shape modes amplitude on the physical parameters above onset.

\section{CONCLUSIONS}

A set of controlled experiments has been conducted to capture the temporal dynamics of nonspherical oscillations of single micrometer-sized air bubbles levitated in water. Shape modes were parametrically excited using a slowly varying amplitude-modulated ultrasonic field. Using two synchronous high-speed cameras located at $90^{\circ}$ provided direct observations of the axisymmetry of the bubble shape, thus validating the modal decomposition of the surface coordinate on zonal harmonics. As the axis of symmetry of the bubble apparent contour corresponds to the orthogonal projection of the bubble axis on the camera view plane, a particular orientation of this axis on at least one camera ensures to correctly define the surface coordinate and to obtain a physically relevant modal decomposition. Once the axisymmetry of the bubble examined in detail, the nonspherical oscillations have been characterized by increasing the acoustic pressure amplitude above the parametric threshold. Several features inherent to the nonlinear behavior of the shape oscillations have been highlighted by the high-speed imaging and successfully compared with a second-order accurate theory. Bubbles exhibiting quadrupolar oscillations show an exponential growth followed by a saturation towards finite amplitude and rapidly induce oscillations of the fourth shape mode through nonlinear coupling. While finite amplitude oscillations are also observed for octupolar bubbles, nonlinear coupling with even modes is not observed for the acoustic pressures considered in the present study. In addition, the order of magnitude of the amplitude of the nonspherical oscillations is recovered by the theory, as well as its dependence on acoustic pressure.

\section{ACKNOWLEDGMENTS}

This work was supported by the French National Research Agency ANR-MOST project CARIBBBOU (Grant No. ANR15-CE19-0003) and the LabEx CeLyA of the University of Lyon (Grants No. ANR-10-LABX-0060 and No. ANR-11IDEX-0007).
[1] T. B. Benjamin and F. Ursell, The stability of the plane free surface of a liquid in vertical periodic motion, Proc. R. Soc. London, Ser. A 225, 505 (1954).

[2] M. S. Plesset, On the stability of fluid flows with spherical symmetry, J. Appl. Phys. 25, 96 (1954).

[3] T. B. Benjamin and M. Strasberg, Excitation of oscillations in the shape of pulsating gas bubbles; theoretical work, J. Acoust. Soc. Am. 30, 697 (1958).

[4] D. Y. Hsieh and M. S. Plesset, Theory of rectified diffusion of mass into gas bubbles, J. Acoust. Soc. Am. 33, 206 (1961).

[5] A. Francescutto and R. Nabergoj, Pulsation amplitude threshold for surface waves on oscillating bubbles, Acust. Acta Acust. 41, 215 (1978).

[6] A. Prosperetti, Viscous effects on perturbed spherical flows, Quart. Appl. Math. 34, 339 (1977).

[7] M. P. Brenner, D. Lohse, and T. F. Dupont, Bubble Shape Oscillations and the Onset of Sonoluminescence, Phys. Rev. Lett. 75, 954 (1995).
[8] S. Hilgenfeldt, D. Lohse, and M. P. Brenner, Phase diagrams for sonoluminescing bubbles, Phys. Fluids 8, 2908 (1996).

[9] Y. Hao and A. Prosperetti, The effect of viscosity on the spherical stability of oscillating gas bubbles, Phys. Fluids 11, 1309 (1999).

[10] A. I. Eller and L. A. Crum, Instability of the motion of a pulsating bubble in a sound field, J. Acoust. Soc. Am. 47, 762 (1970).

[11] Z. C. Feng and L. G. Leal, Energy transfer mechanism in coupled bubble oscillations, Phys. Fluids A 5, 826 (1993).

[12] Z. C. Feng and L. G. Leal, Bifurcation and chaos in shape and volume oscillations of a periodically driven bubble with two-to-one internal resonance, J. Fluid Mech. 226, 209 (1994).

[13] S. J. Shaw, Translation and oscillation of a bubble under axisymmetric deformation, Phys. Fluids 18, 072104 (2006).

[14] S. J. Shaw, The stability of a bubble in a weakly viscous liquid subject to an acoustic traveling wave, Phys. Fluids 21, 022104 (2009). 
[15] A. A. Harkin, T. J. Kaper, and A. Nadim, Energy transfer between the shape and volume modes of a nonspherical gas bubble, Phys. Fluids 25, 062101 (2013).

[16] M. S. Longuet-Higgins, Monopole emission of sound by asymmetric bubble oscillations. Part 1 . Normal modes, J. Fluid Mech. 201, 525 (1989).

[17] M. S. Longuet-Higgins, Monopole emission of sound by asymmetric bubble oscillations. Part 2. An initial-value problem, J. Fluid Mech. 201, 543 (1989).

[18] T. B. Benjamin and A. T. Ellis, Self-propulsion of asymmetrically vibrating bubbles, J. Fluid Mech. 212, 65 (1990).

[19] A. A. Doinikov, Translational motion of a bubble undergoing shape oscillations, J. Fluid Mech. 501, 1 (2004).

[20] M. Strasberg and T. B. Benjamin, Excitation of oscillations in the shape of pulsating gas bubbles; experimental work, J. Acoust. Soc. Am. 30, 697 (1958).

[21] R. G. Holt and D. F. Gaitan, Observation of Stability Boundaries in the Parameter Space of Single Bubble Sonoluminescence, Phys. Rev. Lett. 77, 3791 (1996).

[22] D. F. Gaitan and R. G. Holt, Experimental observations of bubble response and light intensity near the threshold for single bubble sonoluminescence in an air-water system, Phys. Rev. E 59, 5495 (1999).

[23] M. Versluis, D. E. Goertz, P. Palanchon, I. L. Heitman, S. M. van der Meer, B. Dollet, N. de Jong, and D. Lohse, Microbubble shape oscillations excited through ultrasonic parametric driving, Phys. Rev. E 82, 026321 (2010).

[24] T. J. Asaki and P. L. Marston, Free decay of shape oscillations of bubbles acoustically trapped in water and sea water, J. Fluid Mech. 300, 149 (1995).
[25] E. H. Trinh, D. B. Thiessen, and R. G. Holt, Driven and freely decaying nonlinear shape oscillations of drops and bubbles immersed in a liquid: experimental results, J. Fluid Mech. 364, 253 (1998).

[26] E. Trinh, A. Zwern, and T. Wang, An experimental study of small-amplitude drop oscillations in immiscible liquid systems, J. Fluid Mech. 115, 453 (1982).

[27] M. Guédra, C. Inserra, C. Mauger, and B. Gilles, Experimental evidence of nonlinear mode coupling between spherical and nonspherical oscillations of microbubbles, Phys. Rev. E 94, 053115 (2016).

[28] M. Guédra, C. Inserra, B. Gilles, and C. Mauger, 2016 IEEE International Ultrasonics Symposium (IUS) (IEEE, Tours, France, 2016), pp. 1-4.

[29] B. Dollet et al., Nonspherical oscillations of ultrasound contrast agent microbubbles, Ultrasound Med. Biol. 34, 1465 (2008).

[30] H. J. Vos, B. Dollet, M. Versluis, and N. de Jong, Nonspherical shape oscillations of coated microbubbles in contact with a wall, Ultrasound Med. Biol. 37, 935 (2011).

[31] V. Poulichet and V. Garbin, Ultrafast desorption of colloidal particles from fluid interfaces, Proc. Natl. Acad. Sci. USA 112, 5932 (2015).

[32] V. Poulichet, A. Huerre, and V. Garbin, Shape oscillations of particle-coated bubbles and directional particle expulsion, Soft Matter 13, 125 (2017).

[33] For air bubbles of few tens of micrometers in size in water, small values of the Bond number $\mathrm{Bo} \simeq 10^{-4} \ll 1$ allows to infer that effects due to gravity are negligible in our experiments, and that gravity is unlikely to induce a preferential direction for shape deformations. 